\title{
Resources Structure Temporal and Spatial Distribution Characteristics based on Rough Set and Process Neural Network
}

\author{
YanjieYu ${ }^{1, a}$ \\ ${ }^{1}$ School of business and management, Henan Institute of Engineering, Zhengzhou 451191, China \\ ayuyanjie2011yyj@yeah.net
}

Keywords: Human capital, Neural network, Data mining, MATLAB software, Relevance, Rough set.

\begin{abstract}
In order to analyze the spatial and temporal characteristics of the human capital structure data based on rough set and process neural network algorithm, this paper proposes a new data mining method, which can effectively reveal the association temporal and spatial characteristics of human capital output. In order to study the effectiveness and reliability of the algorithm, this study adopts the female human capital output data sample as the research object, and uses the rough set algorithm and process neural network algorithm to do spatial-temporal data mining on the human capital structure data, and uses sequential circuit to do series analysis on data signal. Finally this paper uses MATLAB software to do series computation on data signal, and the associated curve of human capital output is obtained, which provides theoretical reference for data mining on female human capital.
\end{abstract}

\section{Introduction}

For a long term, China implements two elements economic structure, the economic development is inclined to the interests of city, which results in the gap of economic development between city and rural areas. And the obvious gap between urban and rural areas restricts the overall level of economic development in Henan province [1-3]. In order to study the problem of the female human capital output, this paper uses rough set and process neural network to analyze the output of female human capital structure. The calculation process and algorithm design framework is shown in Figure 1.

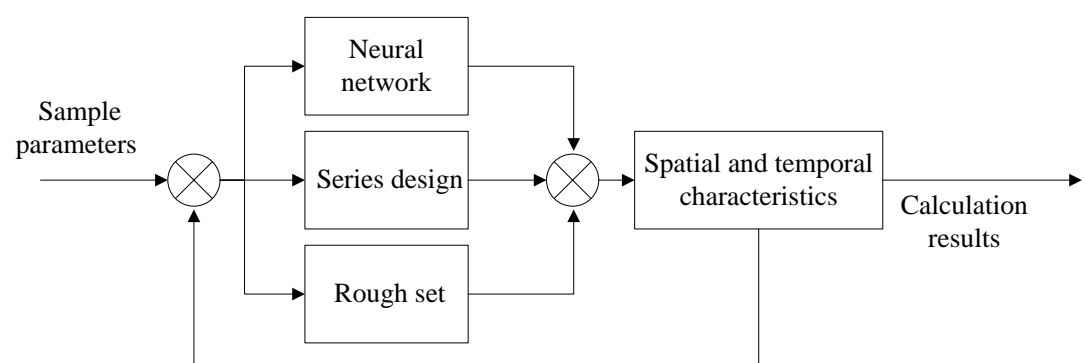

Fig.1: The calculation process and algorithm design framework

As shown in Figure 1, it represents the spatial and temporal characteristics data mining and algorithm design framework. In the analysis process of sample parameters it uses network algorithm and rough set, and gets data temporal and spatial distribution characteristics by using the series parallel design $[4,5]$. Finally the data is feedback to realize the data optimization process.

\section{Spatial-temporal Data Mining Algorithm Design based on Rough Set and Process Neural Network}

Because the samples of female human capital have many cultural levels of population, so the analysis of sample nodes needs the help of process design network [6-8]. The different cultural levels are included in the process neurons hidden layer nodes, and the schematic diagram is as shown in Figure 2. 


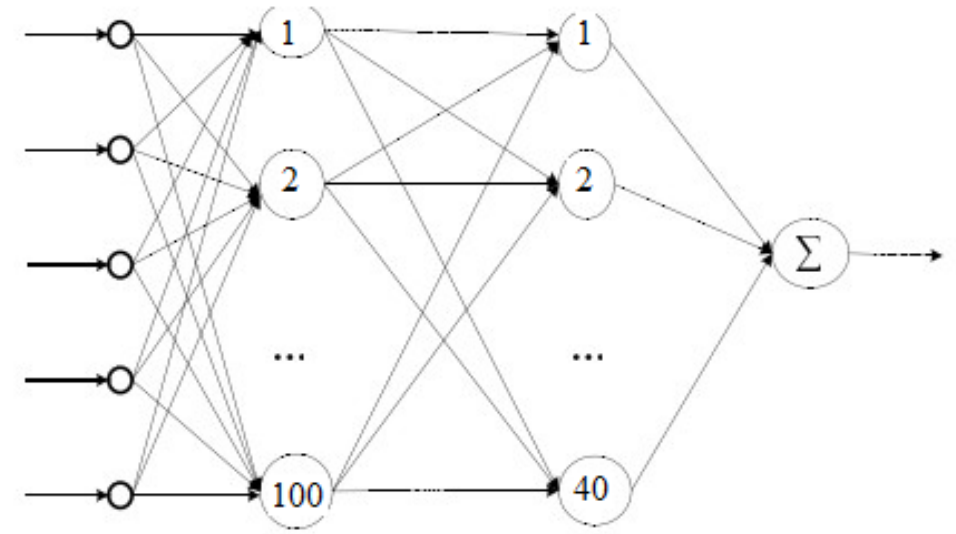

Fig.2: Schematic diagram of process neural network

As shown in Figure 2 it represents process neural network schematic of human capital. The graph has a total of 100 process neurons hidden layer nodes, 40 general neurons hidden layer nodes and 1 output nodes, which is corresponding to the human capital structure spatial distribution structure in the time point. Neural network algorithm commonly uses root mean square error to evaluate the performance of learning, and the expression is as follows:

$$
\mathrm{e}=\sqrt{\frac{\sum_{i=1}^{m} \sum_{j=1}^{n}\left(d_{i j}-L_{i j}\right)}{m^{*} n} .}
$$

Among them, the number of training samples is $m$; the number of the neural network output is $n$; $L_{i j}$ is data sample before the training; $d_{i j}$ is sample data after the training. According to the cost function $X$, it can get the error formula.

$$
\mathrm{e}=\sqrt{\frac{\sum_{i=1}^{m} \sum_{j=1}^{n}\left(d_{i j}-L_{i j}\right)}{X \mathrm{~m}^{*} n} .}
$$

The relationship between cost function and training sample function formula is:

$$
\mathrm{L}=x /\left(1-\frac{1}{\sqrt{x}}\right)
$$

The spatial and temporal distribution characteristics of process neural network are as shown in Figure 3.

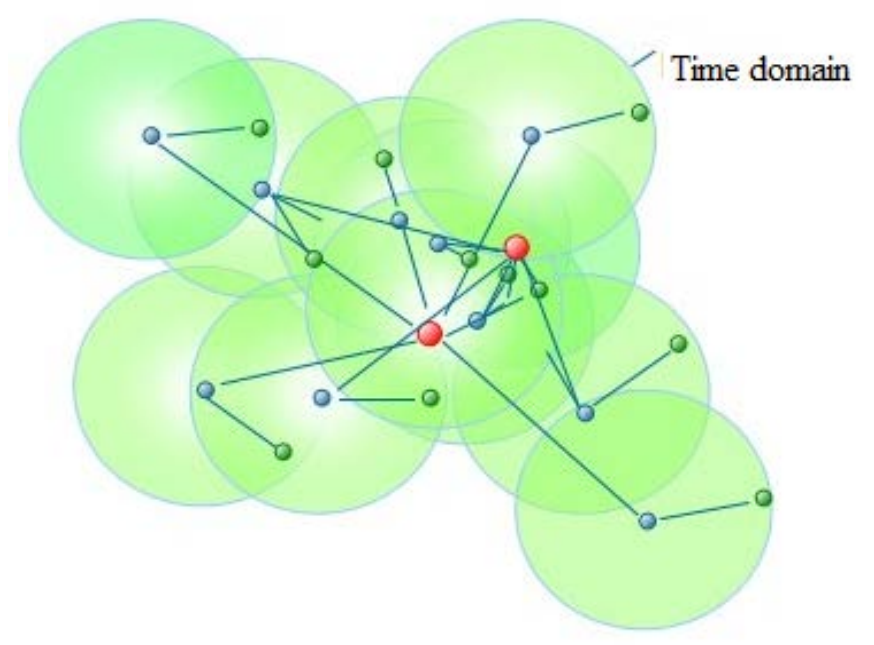

Fig.3: Temporal and spatial distribution space theory

Because the human capital data sample has uncertainty, so sample data boundary is fuzzy. The boundary of human capital rough sets can be expressed as: 


$$
\begin{array}{cccc}
1 & T_{12} & \cdots & T_{1 i} \\
T_{21} & 1 & \cdots & T_{2 i} \\
\vdots & \vdots & \ddots & \vdots \\
T_{j 1} & T_{j 2} & \cdots & 1
\end{array}
$$

The introduction of functional operator is as shown in the formula (5).

$$
\alpha(u, v)=\iint_{G}\left(\frac{\partial u}{\partial x} \frac{\partial v}{\partial x}+\frac{\partial u}{\partial y} \frac{\partial v}{\partial y}\right) d x d y
$$

Among them, $T_{j i}$ is the value of object $j$ according to the certain similarity of object $i$. The bigger the value of $T_{j i}$, the similarity between two objects is smaller. In order to realize the combination of rough set and process neural network, this paper uses MATLAB to realize the series of algorithm, and the main program is as follows:

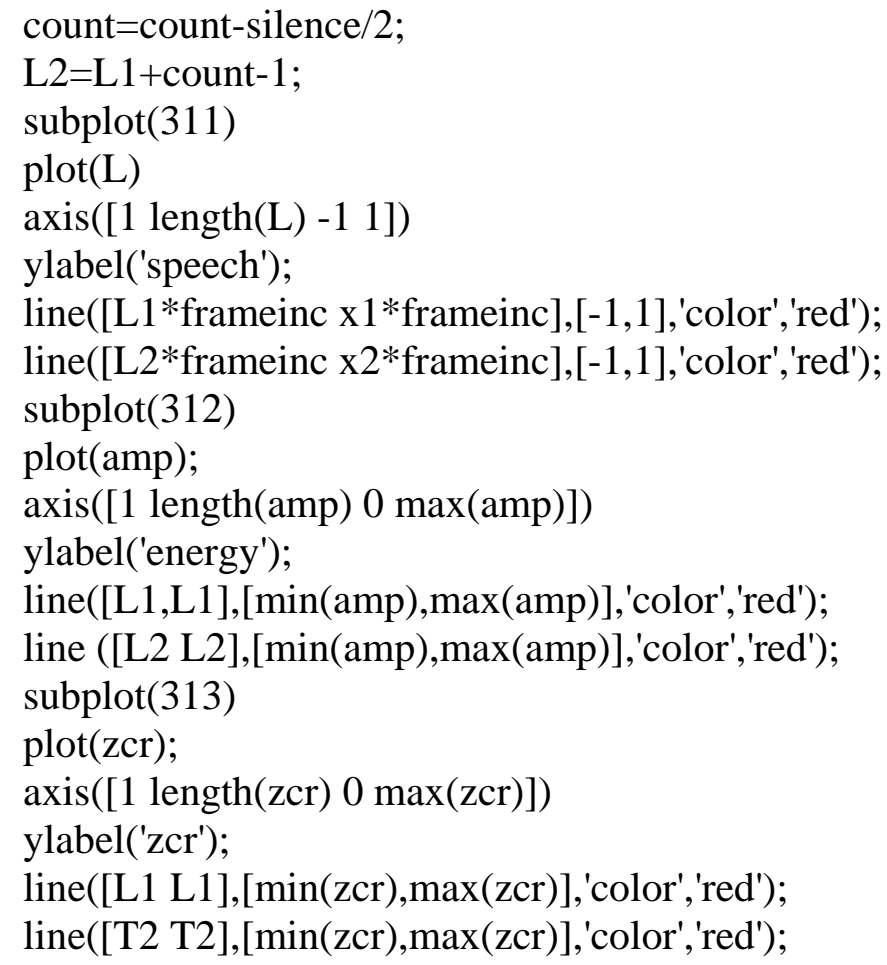

\section{The Spatial and Temporal Distribution Characteristics Analysis of Human Capital Structure Data Mining}

In order to verify the effectiveness and reliability of process neural network and rough set algorithm designed in second section, this study adopts the data structure of female human capital output as the research object, and selects the different cultural level of human capital as the sample [9-11]. The sample data is as shown in Table 1.

Table 1: Human capital data sample

\begin{tabular}{lll}
\hline Education level & Urban women & Rural women \\
\hline Illiteracy & 1053 & 2540 \\
Primary school & 3130 & 7520 \\
Junior high school & 5807 & 12397 \\
High school & 2614 & 1896 \\
College degree and above & 1608 & 264 \\
Above & 14212 & 24617 \\
\hline
\end{tabular}

Table 1 shows the selected human capital data samples in this paper. In order to realize the spatial and temporal characteristics calculation of the sample data, and gets the relevance of sample data 
[12]. It needs to use the time series data series circuit to analyze the sample data signal, and the structure principle of the sequential circuit is as shown in Figure 4.

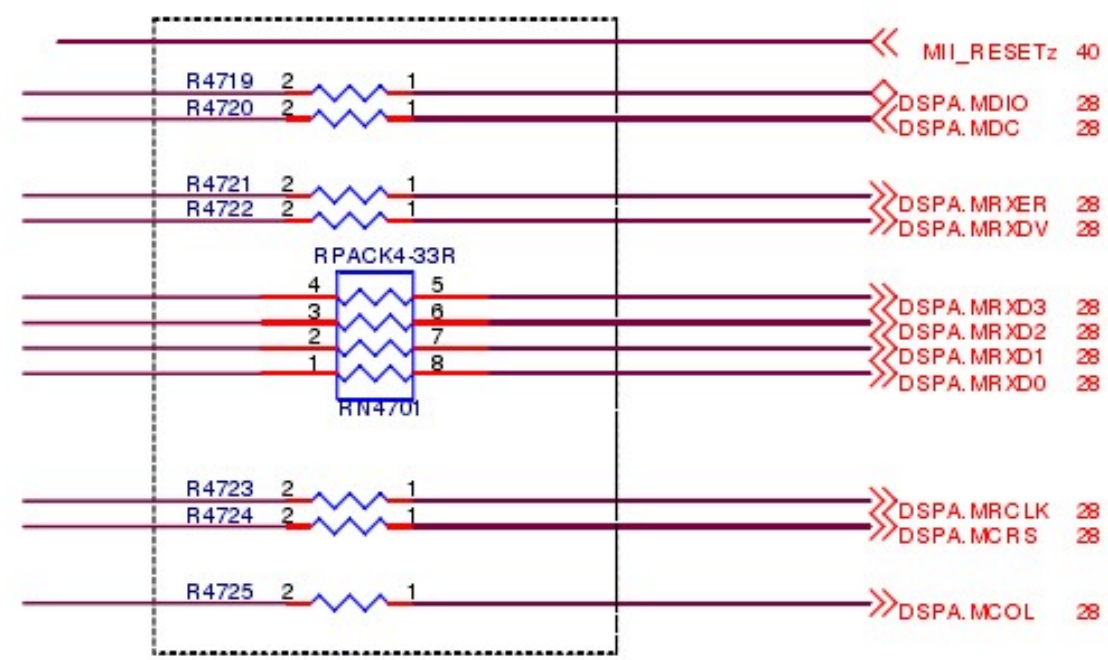

Fig.4: Time series data series circuit

In the process of calculation, human capital $t 1, t 2$ time data and $L 1, L 2$ spatial data have higher data transmission rate, so it needs to increase the necessary termination circuit to ensure the quality of signal transmission. This research uses time series data series circuit structure analysis, and it needs to pay attention to continuous impedance of the high-speed signal [13]. Providing continuous reference reflux path is effective solution to decrease the minimum loop area.

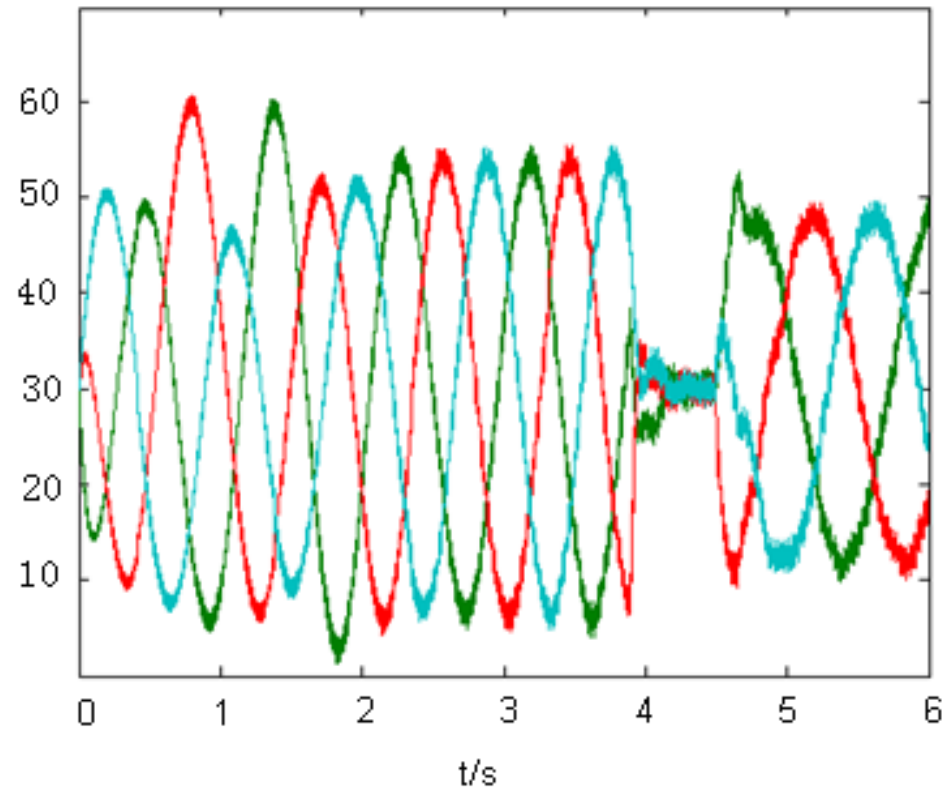

Fig.5: Human capital output data result

Figure 5 shows the human capital production correlation curve by using Simulink toolbox of MATLAB. It mainly includes township human capital output, city human capital output and rural city joint output [14]. And its associated distribution is as shown in Figure 6. 


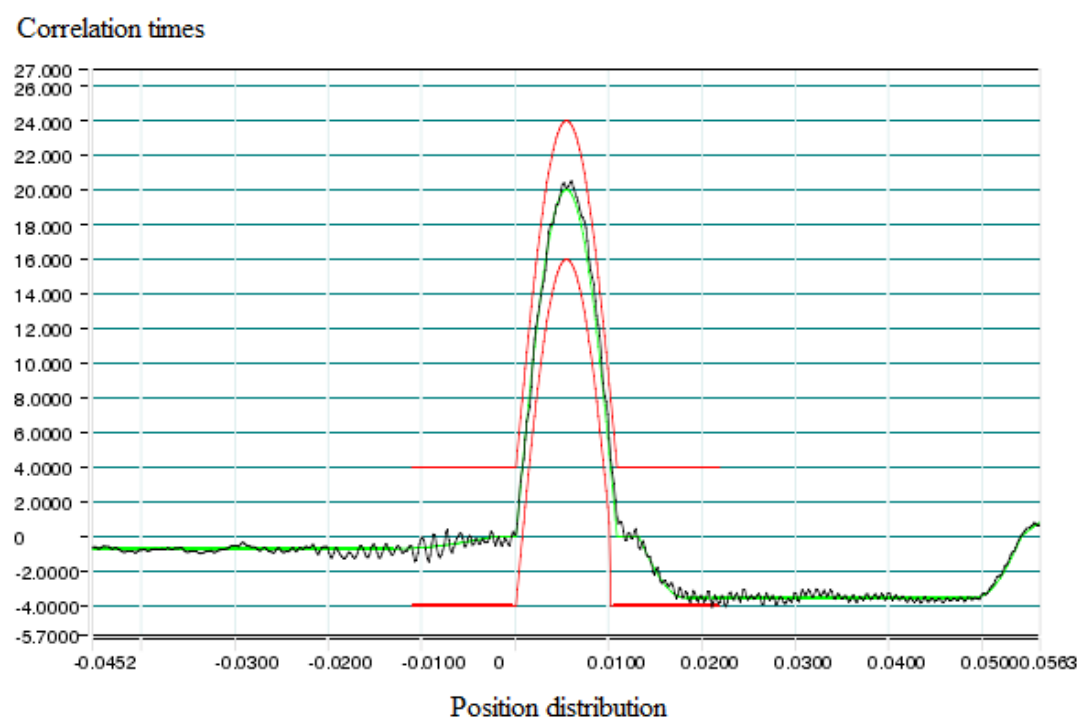

Fig.6: Human capital structure correlation distribution

Figure 6 shows the correlation curve of villages and towns female human capital output. From the chart it can be seen, after the environmental adaptability test, it verifies the environment adaptability of real-time image processing system, which further explains that the system is stable and reliable.

Table 2: The $t_{1}, t_{2}, L_{1}$ and $L_{2}$ correlation analysis after taken the logarithm

\begin{tabular}{llllll}
\hline & & $t_{1}$ & $t_{2}$ & $L_{1}$ & $L_{2}$ \\
\hline \multirow{2}{*}{ LNY } & Pearson correlation & 1 & $0.983^{* *}$ & $0.815^{* *}$ & $0.593^{* *}$ \\
& Significant (bilateral) & & 0.000 & 0.000 & 0.006 \\
\multirow{2}{*}{ LNK } & Pearson correlation & $0.983^{* *}$ & 1 & $0.732^{* *}$ & $0.612^{* *}$ \\
& Significant (bilateral) & 0.000 & & 0.000 & 0.004 \\
\multirow{2}{*}{ LNL1 } & Pearson correlation & $0.815^{* *}$ & $0.732^{* *}$ & 1 & $0.533^{*}$ \\
& Significant (bilateral) & 0.000 & 0.000 & & 0.016 \\
\multirow{2}{*}{ LNL2 } & Pearson correlation & $0.593^{* *}$ & $0.612^{* *}$ & $0.533^{*}$ & 1 \\
& Significant (bilateral) & 0.006 & 0.004 & 0.016 & \\
\hline
\end{tabular}

Note: ** marked significant correlation at $1 \%$ level (bilateral)

* marked significant correlation at 5\% level (bilateral)

From the analysis results, Henan province rural and urban women practitioners have varying degrees of contribution to economic growth. The output elasticity of rural women is higher than output elasticity coefficient of urban working women [15]; this also reflects in the course of development of Henan economy, rural women play a very important role.

\section{Summary}

Based on rough set and process neural network algorithm, this paper presents a new spatial and temporal characteristics data mining, and the method uses fuzzy boundary of rough set and residual training of neural network to do series design on the algorithm, which greatly improves the efficiency of data correlation analysis. In order to study the effectiveness and reliability of the algorithm, this paper uses MATLAB to do series calculation on the algorithm, and obtains the correlation curve of human capital production. Finally it obtains the significant analysis results of the temporal distribution characteristics of $t_{1}$ and $t_{2}$, at the same time the spatial distribution characteristics of $L_{1}$ and $L_{2}$, which provides a new computer method for the human capital production data mining. 


\section{References}

[1] A. Yan, Differences in human capital and regional economic growth trend in China. Huazhong Normal University, 2012: 2-16.

[2] C.H. Liao, Y.Y. Duan. Comparative empirical study of education, human capital on regional growth effect based on the data of Sichuan Chongqing. Economy research, 2013, 4(11): 56-59.

[3] Q.M. Wei. Female entrepreneurship human capital cooperative research and development. Journal of Shanxi University of Finance and Economics, 2012, 4(3): 42-45).

[4] X.L. Tao. Chinese economic growth issue of human capital structure research. Yunnan University, 2012: 2-15.

[5] X. Wen. China women education development report - 30 years of reform and opening up. Social Sciences Academic Press 2012: 40-62.

[6] Z.G. Yang. Human capital investment and the income gap: based on the subject of human capital investment. Ideological front, 2012, 4(5): 92-95.

[7] R. Zhang, X.Z. Lin. Seminar on the rise of central China. Zhongzhou academic journal, 2013, 4(11): 45-48.

[8] C. Zhang, M.L. Liu. The logistics demand of Jiangxi railway based on multiple regression analysis and rough set. Enterprise economy, 2013, 5(6): 56-59.

[9] H.W. Peng, F.R. Liu, X.F. Yang. Prediction of short-term wind power based on artificial neural networks. Journal of solar energy, 2013, 2(8): 52-55.

[10] J.M. Yan. Electric power load forecasting based on trend extrapolation. Chinese foreign capital, 2013, 4(2): 112-115.

[11]X.M. Xu, C.F. Chen. Study on the key problems of time series analysis. Agricultural network information, 2012, 3(3): 78-80.

[12] Y.H. Liu, J.L. Cao. Theory and development trend analysis of time series. Computer knowledge and technology, 2012, 6(1): 12-16.

[13] T.X. Yao, S.F. Liu. Discrete GM model characteristics and optimization. System engineering theory and practice, 2013, 2(4): 25-28.

[14] X. Zhang, J.B. Li, J.X. Zhang. Random missing data time fill method in periodical time series. China health statistics, 2012, 5(2): 46-49.

[15] Akira Yakita. Human capital accumulation, fertility and economic development. Journal of Economics, 2012, 6(4): 1101-1106. 